\title{
ALQIYAM ALTSAQAFIYAH FI ALKITAB ALARABIYAH BAIYNA YADAIK
}

\author{
( ALKITAB ALAWWAL WA TSANI )

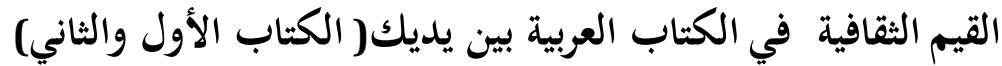

\author{
Mar'atul Munadoroh', Mada Wijaya Kusumah², Ahmad Abdul Razak Al-Khatib ${ }^{\Upsilon}$ \\ ${ }^{1}$ Bachelor Student of STIBA Ar Raayah Sukabumi, Indonesia \\ 2STIBA Ar Raayah Sukabumi, Indonesia
}

Munadiroh93@gmail.com

\begin{abstract}
Culture is one of the important factors in Arabic teaching, since the teaching of language and it's vocabolaries and implications accurately can not take place before the knowledge of culture. This study aims to identify the cultural values in the book Al-Arabiyah Baina Yadaika (book I and II) and it's ways of development. The study was based on the library study because it is appropriate for the purposes of the study. The study found the following results: There are cultural values included in the book Al-Arabiyah Baina Yadaika (book I and II), including: the practice of Islamic greeting in daily life, and earn a living from the permissible professions in Islam, and love of the Prophet aلd and his family, an encouragement to go to Jama'ah prayer and to help each others, an encouragement to choose a good community to live in, and to choose useful hobby, to show attention in traveling behaviors and Islamic magnificance, an encouragetion to use leisure times, and to appreciate the value of the health, and the urge to performe the pillars of Islam, and show attention in the needs of soul, etc. and ways to develop it in the spirit of learners.
\end{abstract}

Keywords: Cultural, Values, Al-Arabiyah Baina Yadaik

\section{مقدمة}

إن اللغة عنصر أساسي من عناصر الثقافة، وهي وعاء الثقافة لأن تعليم اللغة وألفاظها ودلالاتما على نخو دقيق لا

تتم إلا في إطارها الإجتماعي والثقافي، وليس من اليسير تعلم لغة ما دون التعرض لثقافة أصحاها، (قيمهم واتجاهاتم وأنماط

معيشتهم وعقائدهم). والثقافة العربية بعد نزول القرآن الكريع بلغة العرب صارت إسلامية، وأصبحت اللغة العربية لغة تعبدية 
يفرضها الدين الإسلامي أينما حل، ويحملها معه حيثما انتشر، والعربية هي لغة الثقافة الإسلامية بلا نزاع ( عبد الرحمن بن إبراهيم الفوزان، ال 1 • Yم). انطلاقا من هذا تبرز دافعية الباحثة على كتابة هذه الدراسة.

وهذه الدراسة تحت الموضوع "القيم الثقافية في كتاب العربية بين يديك ( الكتاب الأول والثاني )"، تمدفها الباحثة

إلى استخراج القيم الثقافية في كتاب العربية بين يديك ( الكتاب الأول والثاني) ومعرفة عوامل تنميتها.

\section{منهج الدراسة}

اعتمدت هذه الدراسة على منهج الدراسة المكتبية بما يشمل عليه من خطوات علمية. وتتمثل خطوات هذا المنهج في فحص الموقف المشكل، ومن ثم تحديد المشكلة، واختيار أساليب جمع البيانات وإعدادها، وتقنين أساليب جمع البيانات وأخيرا وصف النتائج وتحليلها وتفسيرها. وذلك بهدف وصف الظاهرة موضوع الدراسة وجمع البيانات اللازمة عنها مع فهمها وتحليلها من أجل الوصول إلى المبادئ والقوامين المتصلة بها.

\section{نتائج الدراسة و مناقشتها}

\section{•تعريف الكتاب العربية بين يديك}

زاد الاهتمام في هذا العصر باللغة العربية؛ مما أدى إلى تأليف كتب وسلاسل عديدة، تلبية لحاجات طلاب العربية المتعددة والمتجددة. وبالرغم من الجهود التي بذلت في هذا المحال، فما زالت الحاجة ماسة لسلاسل جديدة، تثري هذا

$$
\text { الحقل المهم (عبد الرحمن بن إبراهيم الفوزان، ع ا • بم). }
$$

تأتي سلسلة العربية بين يديك، إسهاما في هذا الميدان، ومشاركة فيه. وفيما يلي تعريف موجز بأهم ملامح هذه السلسلة: أولا: أهداف السلسلة

تحدف السلسلة إلى تمكين الدارس من الكفايات التالية: الكفاية اللغوية، والكفاية الاتصالية، الكفاية الثقافية.

ثانيا: جمهور السلسلة

السلسلة موجه للدارسين الراشدين، سواء أكانوا دارسين منتظمين في مؤسسات تعليمية، أو دارسين غير منتظمين، يعلمون أنفسهم بأنفسهم، وسواء تم تدريس السلسلة في برنامج مكثف، خصصت له ساعات كثيرة، أو في برنامج غير مكثف خصصت له ساعات قليلة. 
تعتمد السلسلة على اللغة العربية الفصيحة، ولا تستخدم أية لهجة من اللهجات العربية العامية، كما أفها لا تستعين

$$
\text { بلغة وسيطة. }
$$

أولا: لغة

"القيم جمع القيمة، يقال قيمة الشيئ: قدره، وقيمة المتاع: ثمنه، ومن الإنسان طوله، ويقال: ما لفلان قيمة: ماله

$$
\begin{aligned}
& \text { ثياب ودوام على الأمر"(مجمع اللغة العربية، ال ـ بم). } \\
& \text { ثانيا: اصطلاحا }
\end{aligned}
$$

"بحموعة من الأخلاق الفاضلة التي اعتمدت على التربية الإسلامية في توجيه السلوك البشري للقيام بكل عمل،

$$
\text { أو قول يدل على الخير"(بحد حضر، } 7 \text { ا • ب م) }
$$

ت. أهمية القيم

إن القيم تشكل مضمون الثقافة ومحتواها، والثقافة هي التعبير الحي عن القيم، كما أها تلعب دوراً بارزاً في تحديد سلوك الفرد، ويمكن تلخيص أهميتها في الأمور الآتية: • تشكيل الجانب المعنوي في السلوك الإنساني. 
• تشكيل الشخصية الفردية وتحديد أهدافها في إطار معيار صحيح.

• إصلاح الفرد نفسيا وخلقيا، وضبط دوافعه وشهواته ومطامعه كي لا تتغلب على عمله وتوجهه. • دفع الفرد إلى العمل وتوجه نشاطه، وتعمل على حفظ نشاطات الأفراد وبقائها موحدة ومتناسقة.

ث. تعريف الثقافة

أولا: لغة

"الثقافة لغة: مادة (ث ق ف) في اللغة لما دلالات: التقويم، والإدراك، والحذق،يقال: ثقف الشيئ: أقام المعوج،

ومنه: ثقفت الرمح أقمت المعوج منه، وسويته".

ثانيا: اصطلاحا

"بحموعة المعارف والمعلومات النظرية، والخبرات العملية المستمدة من القرآن الكريم والسنة النبوية التي يكتسبها

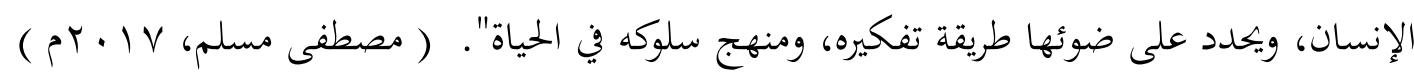

ج. أهمية الثقافة

•تُعتبر الثقافة وسيلة للقضاء على الجهل والتخلف.

•تعد الثقافة من أكثر العوامل تأثيرا في التنمية البشرية.

•دفع الأفراد في البمتمعات للإبداع والتميز من خلال تسيير وتوجيه أفكارهم.

•صقل شخصية الفرد وصهرها في بوتقة الثقافة والتنمية.

•اكساب الفرد التحضر الإنساني والرخاء الفكري.

•الكشف عن نقاط القوة في المحتمع وتحفيزها وتوجيهـا نحو التطور والإصلاح.

•تساهم في تقويم ودعم الحياة الاجتماعية.

•الثقافة وسيلة لمواكبة التطورات والتغيرات التي تطرأ على حياة الأفراد والمجتمع.

$$
\text { •زيادة مستوى الوعي الثقافي الفردي. }
$$


ترتبط القيم بالثقافة ارتباطا وثيقا إذ أن القيم تنبع من ثقافة المجتمع الذي يكون القيم، فلذلك نجد القيم في البحتمعات تختلف باختلاف الثقافات، فنجد تغييرا شاسعا بين القيم الإسلامية و القيم الغربية بسبب الاختلاف الجذذري في الثقافية ومع كون القيم جزءا من الثقافة إلا أها مهيمنة عليه، فالثقافة الحقة لا تخرج عن قيم الجحتمع فنرى قيم المجتمع تحكم ثقافته وتضبطها، لذا لا يمكن فصل القيم عن الثقافة لارتباطهما الوثيق من كافة الجوانب (درداش يامين، 7 ا • بم).

خ. تعريف القيم الثقافية بحموعة عناصر من تاريخ ودين وسياسة نتيجة استقرارها وتفاعلها مع مختلف العناصر المحيطة بها (درداش يامين، $\cdot(\zeta \cdot 1 T / r \cdot 10$

\section{د. عوامل تنمية القيم الثقافية}

إن القيم الثقافية تنمو عن طريق احتكاك الفرد بمواقف متباينة تؤثر عليه بطريقة ما بحيث ينتهي به الأمر إلى تكوين بعض المعايير الخاصة التي تتجمع فتصبح قيما.

\section{وفيما يلي عرض لأهم عوامل تنمية القيم الثقافية:}

\section{•}

إن عناصر الإطار الثقافي من قيم وعادات وأنماط السلوك وغيرها ينقلها الآباء عن الأجداد، ثم تنتقل إلى الأحفاد أو تنتقل عناصر الإطار الثقافي من الكبار إلى الصغار من خلال عملية التنشئة، وهي ما يعرف بعملية التطبيع الاجتماعي.حيث يتم بموجبها اكتساب وتعلم الصغار الطباع والعادات ونماذج السلوك والمعايير وأساليب الحياة والقيم

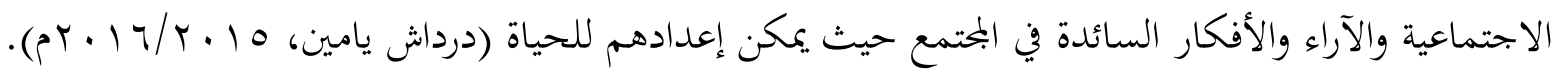

فالأسرة تقوم بإعداد جيل من أجيال المسلمين وتقوم بنقل التراث من جيل إلى جيل آخر من أجل تكوين شخصية ذات القيمة العالية التي تنمو في نفسه القيم الثقافية، لذلك تعتبر الأسرة أها لها دور كبير في غرس وتنمية القيم الثقافية ، وهي وسيلة مهمة وأساسية بين الثقافة والفرد، ومن خلال معايشة الفرد مع أسرته يتحقق غرس وتنمية القيم الثقافية وتغييرها في نفوس الأبناء.

وتعتبر الأسرة كذلك المؤسسة التربوية الأولى التي تتلقى الطفل منذ ولادته، وهي تأخذ دورا رئيسا في غرس البذور الثقافية الأولى في نفوس الطفل، ففيها يقتضي الطفل حياته الأولى ومعها يتفاعل، ومنها يتعلم كيف يتعامل مع نفسه ومع 
الآخرين، ومن خحلالها يكتسب الطفل قيمه واتحاهات وثقافاته وتراثه الاجتماعي ( عبد الكريم منصور ناصر قشلان،

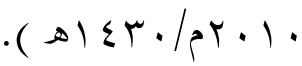

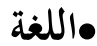

تعتبر اللغة من بين العوامل المساعدة على اكتساب القيم، وذلك باعتبار أن اللغة هي الحامل للقيم التي تسود البحتمع، وأن عملية التعلم تتم بواسطتها. ومن جهة أخرى فاللغة تسهل عملية التواصل والتبليغ بين أفراد المجتمع، ومن المؤكد أن عملية الاتصال في ميدان التنظم والإدارة تعتبر أساسية لحسن العمل، ولففع مستوى فعالية الاتصال في التنظيم، وتأتي أهمية اللغة من حيث أهما تعكس شخصية الفرد وثقافة بحتمع هوأفها تساعد على تعديل الشخصية والثقافة (درداش

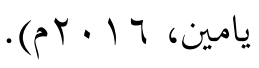

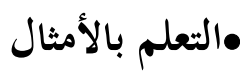

الأمثال من الأساليب التي تقوم بدور فعال في التأثير على تنمية القيم الثقافية لدى المتعلمين وذلك من خلال توضيح

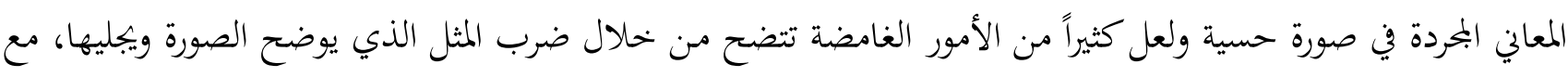
العلم أن الأمثال موجودة في التراث الشعبي للأمم والشعوب، فهي تنتقل من جيل إلى جيل، بحيث تقوم بتلخيص الخبرة

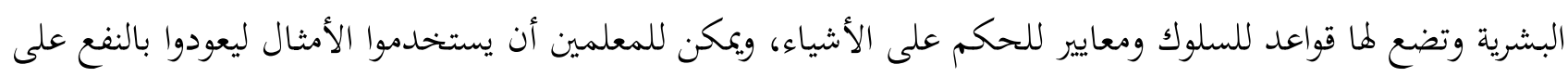
المتعلمين من خلال تقديم معلومات أو مفاهيم جديدة تسهم في غرس قيم إيجابية نافعة وتنميتها، أو تعديل سلوكيات سلبية

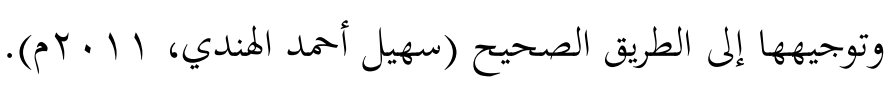

ويمكن أن يقوم المعلم داخل الفصل بتنمية بعض القيم في نفوس المتعلمين من خلال ضرب الأمثال سواء من القرآن

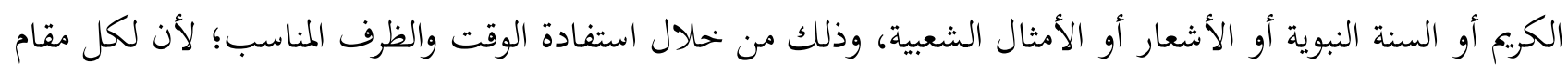
مقال. فمثلا لو أراد المعلم أن يغرس بحموعة من القيم الثقافية في نفوس المتعلمين يمكن المدرس أن يضرب أمثلة من واقع

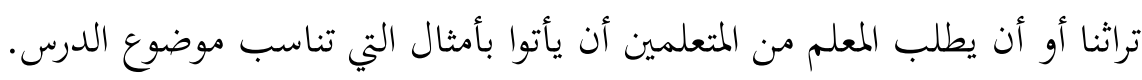

$$
\text { ذ. القيم الثقافية في كتاب العربية بين يديك }
$$

$$
\text { •الحث على ممارسة التحية الإسلامية في الحياة اليومية }
$$

في الحوارات تحت الموضوع التحية والتعارف من كتاب الأول (القسم الأول) صفحة ب و ـ توجد القيمة الثقافية وهي ممارسة التحية الإسلامية في التعارف، حيث أن التعارف في تلك الحوارات تيدأ بالتحية الإسلامية والرد 


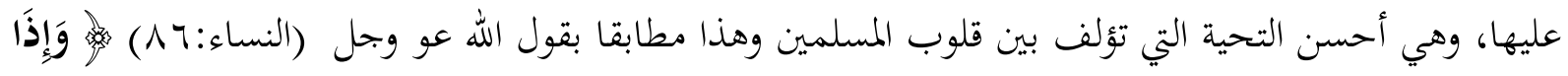

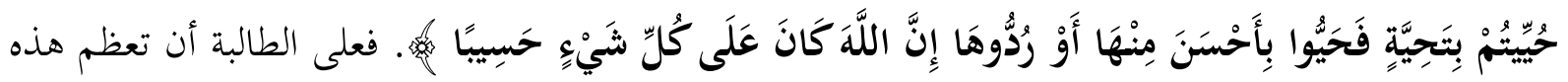
التحية الإسلامسة لأن تعظيمها جزء من تعظيم دين الإسلام.

$$
\text { • (كسب الرزق من المهن المباحة في الإسلام }
$$

في الصفحة ب ميت الموضوع الأسرة من كتاب الأول (القسم الأول) توجد القيمة الثقاقية من خلال الحورات وهي معرفة بعض المهن المباحة في دين الإسلام، فعلى المسلم أن يسع إلى كسب الحلال بطريقة اختيار المهنة

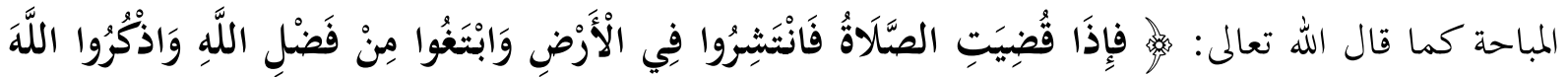

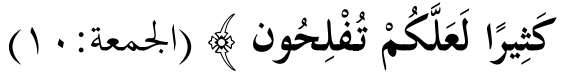

$$
\begin{aligned}
& \text { •حبة الرسول عليهوسللم وأسرته }
\end{aligned}
$$

في الكتاب الأول (القسم الأول) في الصفحة •ـ تحت الموضوع "الأسرة" وجدت القيمة الثقافية وهي معرفة أسرة الرسول عليمولسلمه، فإن معرفتها أمر ضروري حيث أن كلما زاد معرفة الشخص عن شيئ زاد محبته فيه. فعلى كل مسلم أن يهتم هذذا الأمر لأن هؤلاء قدوتنا وأسوتنا، وبمعرفتهم ستزداد العبر والخبرات من قصصطم. •الحث على صلاة الجماعة والبادرة إليها

الحوار في الصفحة بr في الكتاب الأول (القسم الأول) تحت الموضوع "الأسرة" تصور لنا موفق المسلم عند سماع

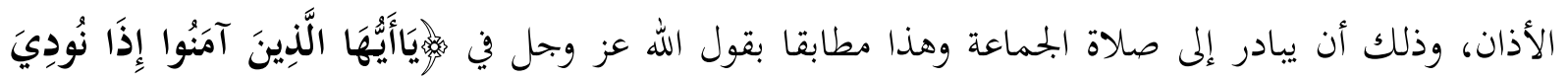

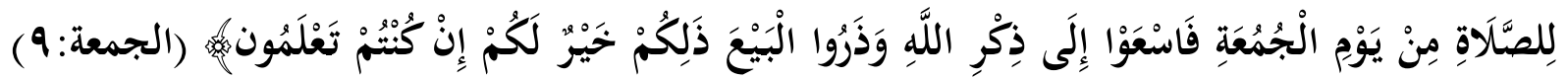

$$
\text { •الحينغي على كل مسلم أن يحث نفسه وغيره على التنافس في الخيرات. }
$$

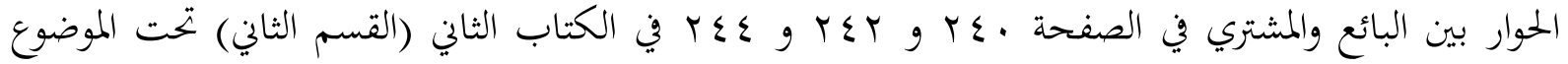
"التسوق" تصور لنا موقف المسلم حيث أنه يبادر إلى مساعدة الآخرين، وهذا الأمر له دور كبير في تأليف بين قلوب المسلمين، فينبغي على كل مسلم أن يبادر إلى مساعدة الآخرين تطبيقا لقول الرسول عليهولسلمه : ( إن الله في

$$
\text { عون العبد مادام العبد في عون أخيه ). }
$$




\section{•الحث على اختيار الهواية المفيدة}

في الصفحة • ب r و r r و و צr في الكتاب الثاني (القسم الأول) تحت الموضوع " الهوايات" توجد القيمة التي ترشدنا إلى اختيار الهواية المفيدة استغلالا للوقت، تطويرا للمهارة و رفعا للمستوى العلمي، ومن الهوايات المفيدة منها: القراءة، كتابة النشرة العلمية، الرماية، ركوب الخيل، السباحة، وغير ذلك، فهذا ما ينبغي على كل مسلم فعله. •تقدير قيمة الصحة في الصفحة r و و و 1ا تحت الموضوع " العناية بالصحة " في الكتاب الأول (القسم الثاني) توجد القيمة التي ترشدنا إلى تقدير قيمة الصحة حيث أن الصحة نعمة عظيمة ومغبون فيها كثير من الناس استدلالا بقول الرسول عليهوسلم : ( نعمتان مغبون فيهنا كثير من الناس الصحة والفراغ ). فينبغي على كل مسلم أن تشكر على هذه النعمة وتهتم بها.

\section{الخلاصة}

توصلت هذه الدراسة أن في الكتاب العربية بين يديك (الكتاب الأول والثاني) تتضمن فيه القيم الثقافية أكثر من ها قيمة، ولكن اختصرت هذه الدراسة بذكر أهمها ومنها: الحث على ممارسة التحية الإسلامية في الحياة اليومية، وكسب الرزق من المهن المباحة في الإسلام، وحمبة الرسول عليهوللم وأسرته، والحثث على صلاة الجماعة والبادرة إليها، والحثث على مساعدة الآخرين، والحث على اختيار الهواية المفيدة، تقدير قيمة الصحة. وكذلك تحديد أهم عوامل تنمية القيم الثقافية منها: الأسرة، واللغة، والتعلم بالأمثال.

\section{قائمة المراجع}

$$
\text { ابن منظور، ج. ( § § اهـ). لسان العوب. بيروت: دار الصادر. }
$$

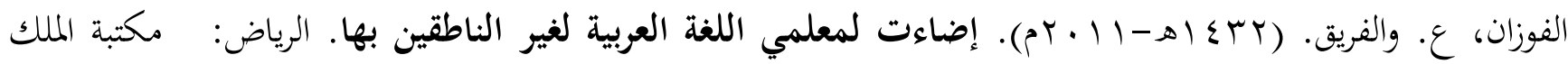

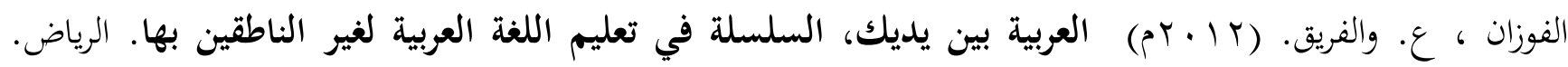
المملكة العربية السعودية، الرياض: العربية للجميع. بحمع اللغة العربية. المعجم الوسيط. القاهرة: دار الدعوة.

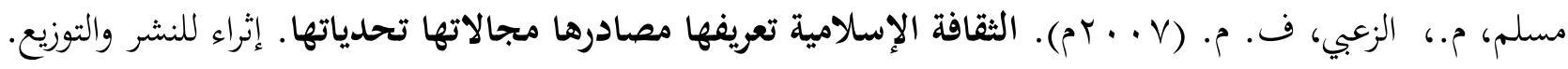




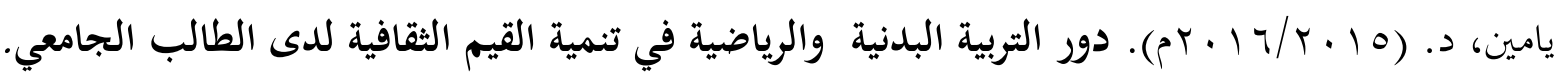
الهندي، س. أ. ( ا . . rم). دور المعلم في تنمية بعض القيم الاجتماعية لدى طلبة الصف الثاني عشر بمحافظات

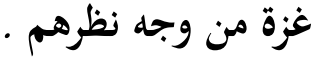

